\title{
A Universal High Impedance Fault Detection Technique for Distribution System Using S-Transform and Pattern Recognition
}

\author{
Manohar Mishra $^{1} \cdot$ Pallavi Routray $^{1} \cdot$ Pravat kumar Rout $^{1}$
}

Received: 19 February 2016 / Accepted: 25 July 2016 / Published online: 5 August 2016

(C) Springer Science+Business Media Singapore 2016

\begin{abstract}
This paper describes a new method for high impedance fault (HIF) detection based on s-transform (ST) and pattern recognition technique. Conventional distance, over current and ground fault relays are difficult to apply for High Impedance Fault (HIF) detection in distribution line because of sensitivity, diversity, selectivity issues in case of low value of fault current. Recently, s-transform has been successfully applied for different power system protection problem. It is a very useful tool to analyze transient fault signal to provide both time and frequency information unlike Fourier transform and the same has been considered for high impedance fault detection in this work. The features extracted using s-transform to train and test the two different intelligent classifier like artificial neural network (ANN) and support vector machine (SVM) separately, to discriminate the HIF with other transient phenomenon (Load switching, capacitor Switching) and also normal fault condition. A comparative study of these two classifiers has been reported based on their detection accuracy. It has been found that the proposed techniques are highly effective for high impedance fault detection under a wide range of operating conditions and noisy environment in a high voltage distribution
\end{abstract}

Manohar Mishra

manohar2006mishra@gmail.com

Pallavi Routray

sonypallavir@gmail.com

Pravat kumar Rout

pkrout_india@yahoo.com

1 Electrical and Electronics Engineering Department, S 'O' A University, Bhubaneswar Odisha, India network. The proposed scheme is fully simulated and analyzed by MATLAB/SIMULINK environment.

Keywords Artificial neural network - High impedance fault $\cdot \mathrm{S}$-transform $\cdot$ SVM

\section{Introduction}

High Impedance Faults (HIFs) generally appear on the primary distribution system when a bare energized overhead conductor comes in contact with high impedance surfaces [1]. HIF current contain some complex characteristics such as asymmetry, nonlinearity and low frequency. Due to that, it is quite challenging for any traditional over-current relays to detect the HIF because unlike other types of faults HIFs give rise to a very small amount of fault current, i.e. in between 10-100 (A) depending on the type of surfaces ( i.e. dry or wet sand, asphalt, sod, grass, trees etc.). The failure of HIF detection may lead to fire hazard and arcing [1]. So it is necessary to identify the HIF and to isolate the corresponding feeder.

The main objective of many detection schemes is to identify the special features in the patterns of voltage and current associated with HIF. Identification techniques generally contain two vital steps: feature extraction and classification [2]. For many years, some protection engineers and researchers had introduced numerous feature extraction and classification techniques such as digital signal processing [3], fractal analysis [4], wavelet transform (WT) [5-10], crest factor [11], coefficient of variation [11], mathematical morphology [12], Kalman filtering [13], decision tree [14] etc. and various classifiers have been used such as Bays, nearest neighbor rule (NNR), artificial neural network (ANN) [15], ANFIS (adaptive neuro-fuzzy inference 
system) [16] etc. One of the main drawbacks of fourier transform (FT) based technique is that it does not have any time information associated with the fault instant. To overcome this limitation of FT another detection scheme based on wavelet transform (WT) was proposed. Unlike FT, the WT possesses information both in the time domain and in frequency domain and it deals with non-stationary signals such as those experiencing HIF arcing fault. The efficiency of WT decreases in noisy environments where as Stackwell transform(ST) [17] is very much sensitive to noise (i.e. it has the ability to identify any type of disturbance correctly in the presence of noise) due to which ST is more effective than WT for detecting HIF in the system. S-Transform has various applications in the field of Geophysics, Genomic Signal Processing, Biomedical Engineering, and Power System Protection.

In this paper, a contingent method is proposed that uses ST for feature extraction. The ST is an extended version of WT which is used to extract the time and frequency components of the voltage and current. The fixed resolution of the short time fourier transform (STFT) and the absence of phase information in the WT lead to the development of the S-transform, which not only retains the absolute phase information but also has good time frequency resolution for all frequencies. Again, this paper analyzes the usefulness of applying an artificial neural network (ANN) and support vector machine (SVM) for HIF classification based on the ST feature vector. The ANN classifiers perform better than other conventional method as it based on the gradient decent method which minimises the total squared error of the output. On the other hand SVM classifier is very much applied in various fields of engineering as it tries to find an optimal hyperplane that maximizes the margin between data samples in two classes in a higher dimensional feature space derived from the original data space through a kernel function. As, both the classifier have different advantages with each other and successfully applied by different researchers for various applications like power quality events classification, transmission line fault classification, speech recognition and islanding classification in microgrid etc. the same has been considered as a classifier for this work.

After capturing the discrete value of voltage and current waveforms for a definite sample of the power system, these data sets aree analyzed by ST and different distinguish features are extracted from STA matrix. Then the extracted features were fed as an input to the classifier to determine whether the HIF occur or not.

The major contributions of this study towards HIF detection problem are: (1) application of ST for feature extraction instead of using other popular signal processing technique like WT and ST. (2) use of SVM and ANN for classification and detection of HIF condition. (3) analysing the application of the above techniques under practical operating conditions. (4) presentation of extensive comparative results with recently published and well proved techniques.

The rest part of the paper structure is organized as follows: The complete power system model and description are introduced in "System Under Study". The brief explanation of the ST is presented in "Stackwell Transform (ST)". A brief description of the proposed classifier is illustrated in "Classification of Events". Results have been illustrated and analyzed in "Results and Discussion" by the proposed methodology which justifies the efficiency of the methods for real time application. Lastly, the conclusion and future work has been enumerated in "Conclusion" and 7 respectively.

\section{System Under Study}

The initial step of every observation is to develop a proper model. In this paper not only HIF model, but also various transient conditions are simulated such as capacitor switch, load switch, normal ground fault models etc. All these models are generated with the help of the power system block set and are sampled at a rate of $5 \mathrm{KHz}$ frequency, which is equal to 100 samples/cycle. The power system fundamental frequency is taken as $50 \mathrm{~Hz}$.

(a) Power system model description with Single Line Diagram:

In the proposed study both radial and mesh distribution system are taken into consideration as shown in the Fig. 1a and $\mathrm{b}$ respectively. In radial distribution system a generator of $50 \mathrm{MVA}$ is connected to supply $138 \mathrm{KV}$ of voltage to the utility sector through a transmission line of $100 \mathrm{~km}$ long and a 138/25 KV star/delta transformer. A HIF fault is created on one of its distribution feeders at $15 \mathrm{~km}$ from its source side. A three branched radial distribution network has been used with both linear and non-linear loads of 4MVA and 1MVA respectively. Two other branches with 2MVA and 4.262MVA non-linear loads are integrated in the system with $0.8 \mathrm{pf}$.

Similarly, in mesh distribution system three generators, each with rating of $50 \mathrm{MVA}$ are used to supply $138 \mathrm{KV}$ of voltage to the utility sector through a $25 \mathrm{KV}$ transmission line. At each end three star-delta transformers are used with the rating of 5MVA to step-down the voltage from $138 \mathrm{KV}$ to $25 \mathrm{KV}$. All other system conditions such as capacitor switching, load switching and normal fault condition has been implemented in the system. In total 4000 cases are used to test for classification purpose. By changing the parameters, 250 cases are taken for each type of faults such as L-G, 
Fig. 1 System Models a Radial System Model (System 1), b

Mesh System Model (System 2)
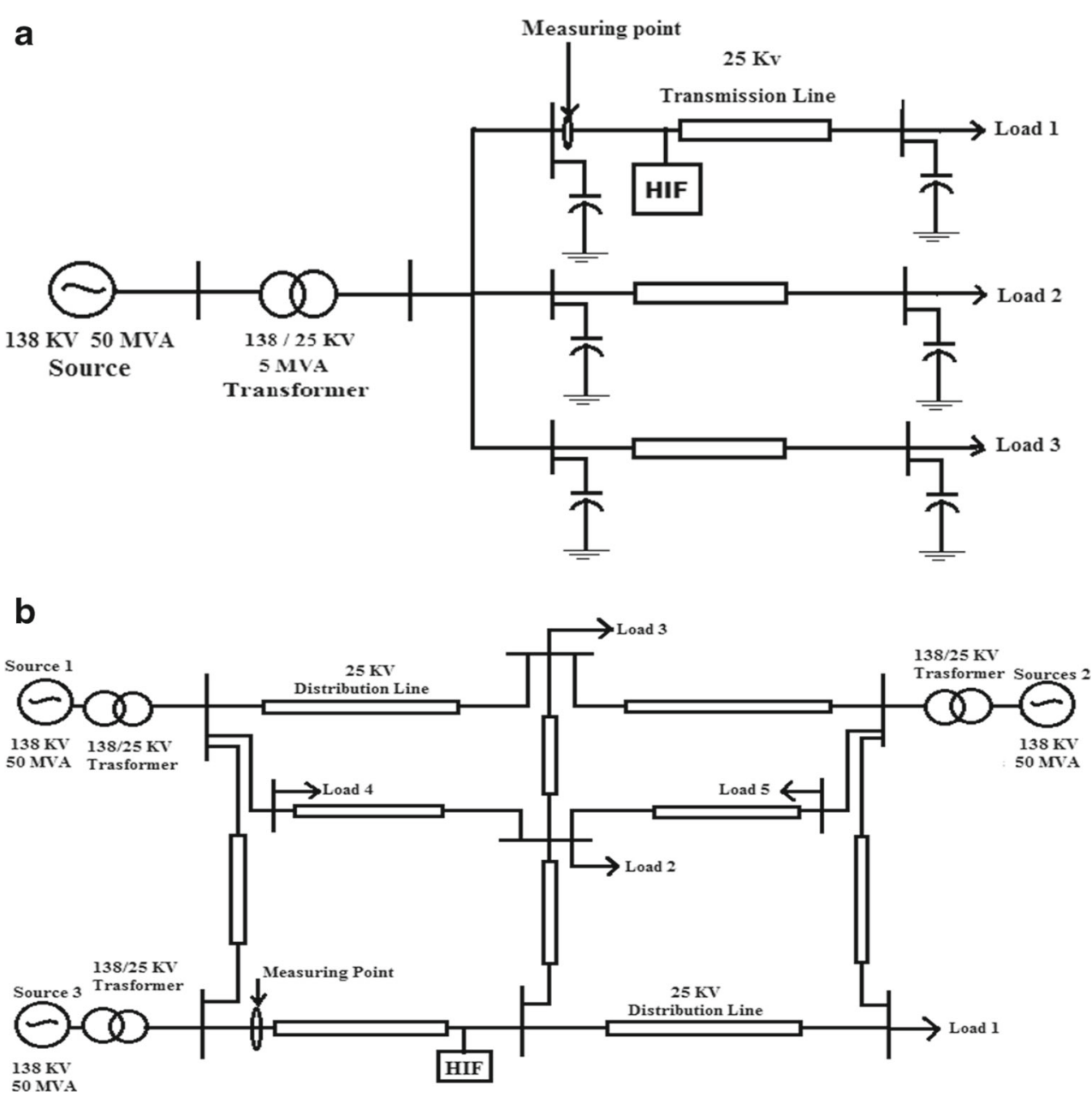

L-L-G, L-L-L-G faults. So a total of 1750 each for HIF and normal fault conditions are simulated. Each with 250 cases of capacitor switching and load switching have been considered and simulated by changing parameters within a certain range.

\section{(b) HIF Model Simulation:}

There are various types of HIF models as proposed by many researchers [18]. Out of which two types of
HIF models are being used in the paper as shown in Fig. 2a and $b$ respectively. Figure 2a shows a simplified HIF model having two unequal resistances that make unsymmetrical fault current [19].

Figure $2 \mathrm{~b}$ shows a simplified two diode HIF model with two anti-parallel diodes (Dp, Dn), two unequal value resistors $(\mathrm{Rp}, \mathrm{Rn})$ which allows asymmetric fault current and
Fig. 2 HIF Model a Type I, b Type II
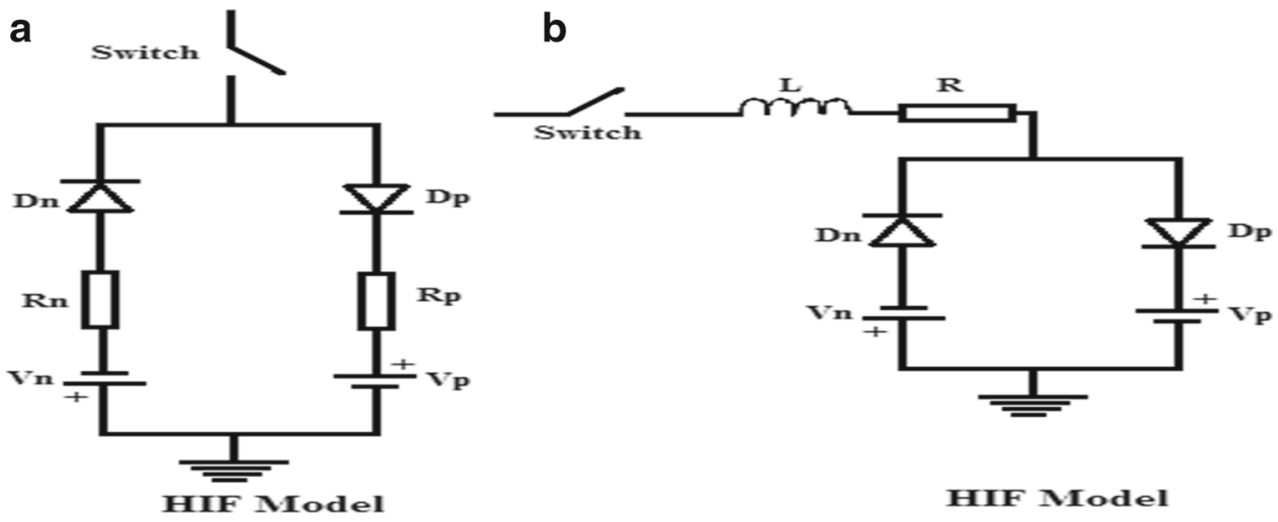

HIF MTodel 

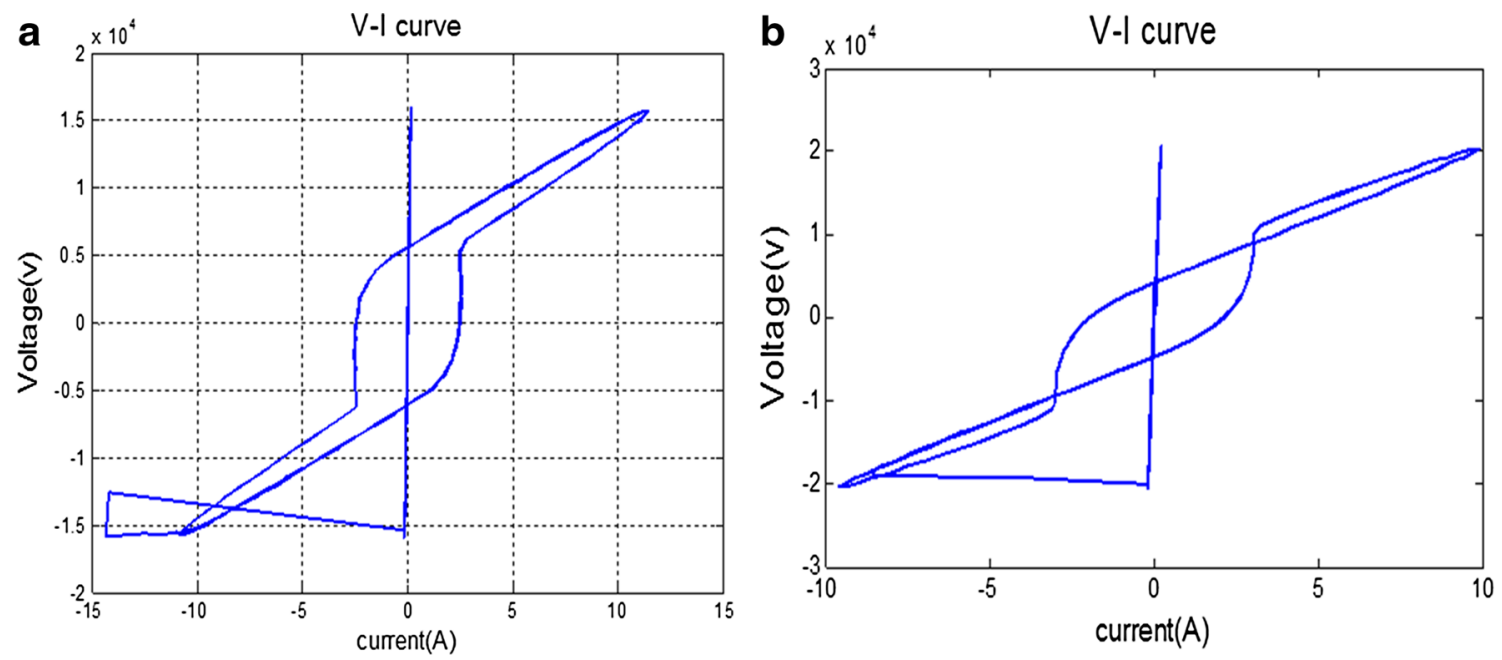

Fig. 3 Simulated HIF V-I Characteristic curve with HIF model Type I and Type II in (a) and (b) respectively

two DC voltage (Vp, Vn) which perform as the inception voltage of air in soil or between trees and distribution line [20]. If $\mathrm{Vph}>\mathrm{Vp}$ then current (I) flows towards the ground and when $\mathrm{Vph}<\mathrm{Vn}$ then flow of current get reversed. For $\mathrm{Vn}<\mathrm{Vph}<\mathrm{Vp}$ no fault current flows. Here, both the models are based on Emanuel arc model. In this paper, both the models are used at different places on the transmission line to simulate the fault current.

Figure $3 \mathrm{a}$ and $\mathrm{b}$ depict the V-I characteristic curve which shows a non-linear cyclic pattern due to HIF model Type I and Type II respectively. Due to the occurrence of HIF at $0.1 \mathrm{sec}$ there is a distortion in current and voltage waveform as shown in Fig. 4a. Figure $4 \mathrm{~b}$ shows the total current and voltage at the sub-station for HIF for single line to ground fault at B-ph.
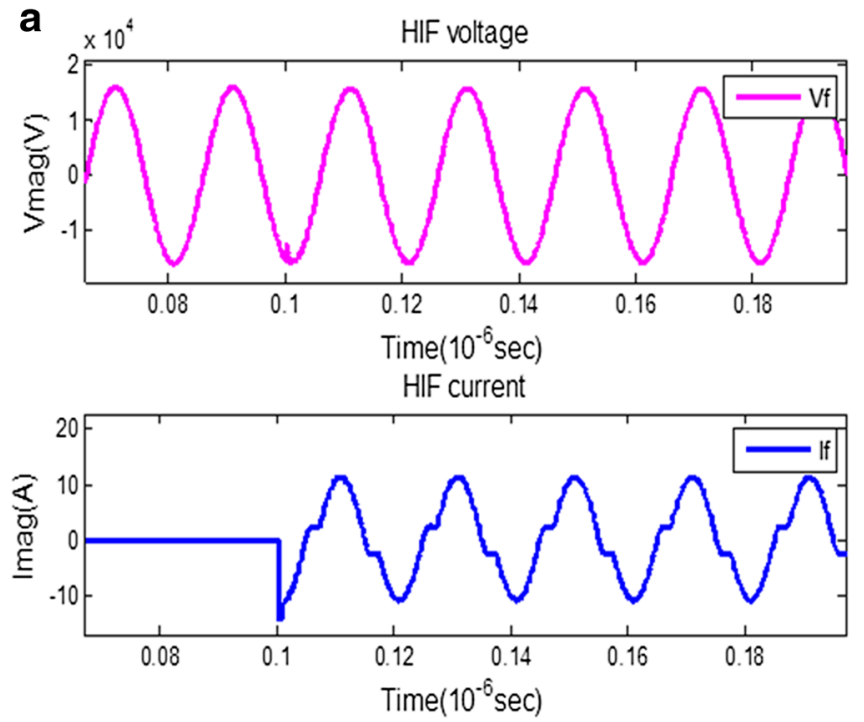

\section{Stackwell Transform (ST)}

The S-transform [20-23] is an extended version of wavelet transform which is based on shifting and scalable localizing Gaussian window. The continuous wavelet transform can be represented as:

$c w t_{g}^{\phi}(\tau, d)=\int_{-\infty}^{\infty} g(t) . \phi(t-\tau, d) d t$

Where the input signal $\mathrm{g}(\mathrm{t})$ is represented as a function of time $(\mathrm{t})$.

The width (d) and the time of spectral localization $(\tau)$ controls the resolution of the wavelet CWT $(\tau, \mathrm{d})$, which is a scaled copy of the fundamental mother wavelet.
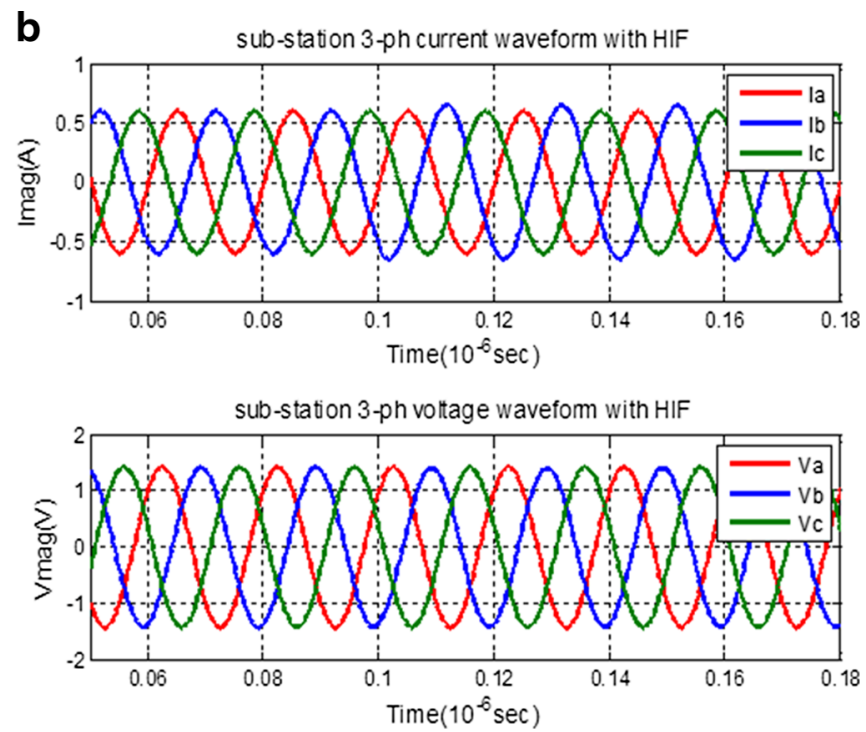

Fig. 4 a Simulated HIF Voltage and current Waveform, b Sub-station current and voltage with HIF at B-g 
Mathematically ST is represented as a CWT along with a précised mother wavelet multiplied with phase factor $\left(e^{-j 2 \pi f t}\right)$.

$S(\tau, f)=e^{-j 2 \pi f t} C W T(\tau, d)$

Where the mother wavelet for the particular case can be represented as

$\phi(t, f)=\frac{|f|}{\sqrt{2 \pi}} \cdot e^{\frac{-t^{2} f^{2}}{2}} \cdot e^{-j 2 \pi f t}$

$\sigma(f)=\frac{1}{|f|}$, is the width of the Gaussian window. The time scale representation is produced by WT while ST is used to produce both time and frequency representation.

The ST may be represented as

$S(\tau, f)=\int_{-\infty}^{\infty} g(t) \times \frac{|f|}{\sqrt{2 \pi}} e^{-\left((t-\tau)^{2} f^{2} / 2\right)} \times e^{j 2 \pi f t} d t$

In discrete form ST may be written as

$S(i, n)=\sum_{m=0}^{N-1} g(n+m) e^{\left(-2 k^{2} \pi^{2} m^{2} / a+b \sqrt{|f|}\right)^{2}} e^{j(2 \pi m i) / N}$

Where $\mathrm{i}=1,2, \ldots \mathrm{N}-1$ and $\mathrm{n}=0,1 \ldots \mathrm{N}-1$ represents as time in sample and frequency step respectively. The output of ST is a $\mathrm{N}^{*} \mathrm{M}$ matrix represented as STA matrix

\section{Classification of Events}

In this section the intelligent classifiers used for the HIF detection are discussed.

\section{Neural Network (NN)}

The Back Propagation Neural (BPN) Network is used as a classifier between HIF and non-HIF events because of its fast response, lesser complexity and flexibility of use. BPN is a multilayer fast forward network with extended gradientdescent based delta-learning rule usually known as back propagation or error rule. Back propagation provides a computationally efficient method for changing the weight in a feed forward network, with differentiable activation function units, to learn a training set of input-output examples [24]. GE Hinton, Rumelhard and R.O.Williams first introduced BPN in 1986.Being a gradient descent method, which minimizes the total squared error of the output computed by the net. The network is trained by supervised learning method $[25,26]$. The architecture of the neural network is shown in Fig. 5.

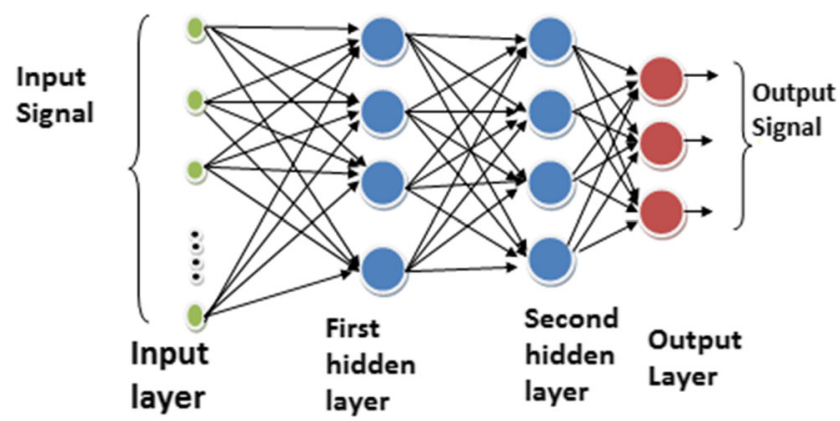

Fig. 5 Architecture of NN

\section{Support Vector Machines (SVM)}

Support Vector Machine is a very important classifier which is widely used in various power system related problems. SVM introduces a unique training algorithm which is used to maximize the boundary between the various classes [27]. It carries a couple of data set: input vector and class ( $\mathrm{Pi}$, Qi), where $i=1,2, \ldots[28]$.

$L(w, \xi)=0.5\left(w^{T} w\right)+C \sum_{i=1}^{l} \xi_{i}, \xi_{i}>0$

$Q_{i}\left(w^{T} \emptyset\left(p_{i}\right)+b\right) \geq 1-\xi_{i}$

The solution of SVM is derived by minimizing Eq. 6 with respect to Eq. 7. Here b, $\omega$ and $\xi$ are the bias, weight and error term respectively. $L$ is known as the penalty factor. The different kernel functions like linear, radial, polynomial, sigmoid function are usually used for mapping and in this study sigmoid function is used due to its better performance. SVM maps the input vector into a large dimensional space. A hyper plane in feature space is used through various mapping functions to increase its ability of classification.

\section{Results and Discussion}

\section{Performance of S-Transform on HIF Fault Detection Visually}

In this paper, HIF, normal faults and other transient signals as capacitor switching and load switching events are taken for analysis. The ST is applied to the entire above mentioned distorted signal to generate time-frequency, time-amplitude and amplitude-frequency plots, that presents the disturbance pattern for visual inspections and to know the nature of the disturbance. All three important information as amplitude, frequency, and time can be easily calculated to detect and classify various signals. These signals are simulated using MATLAB/SIMULINK. 

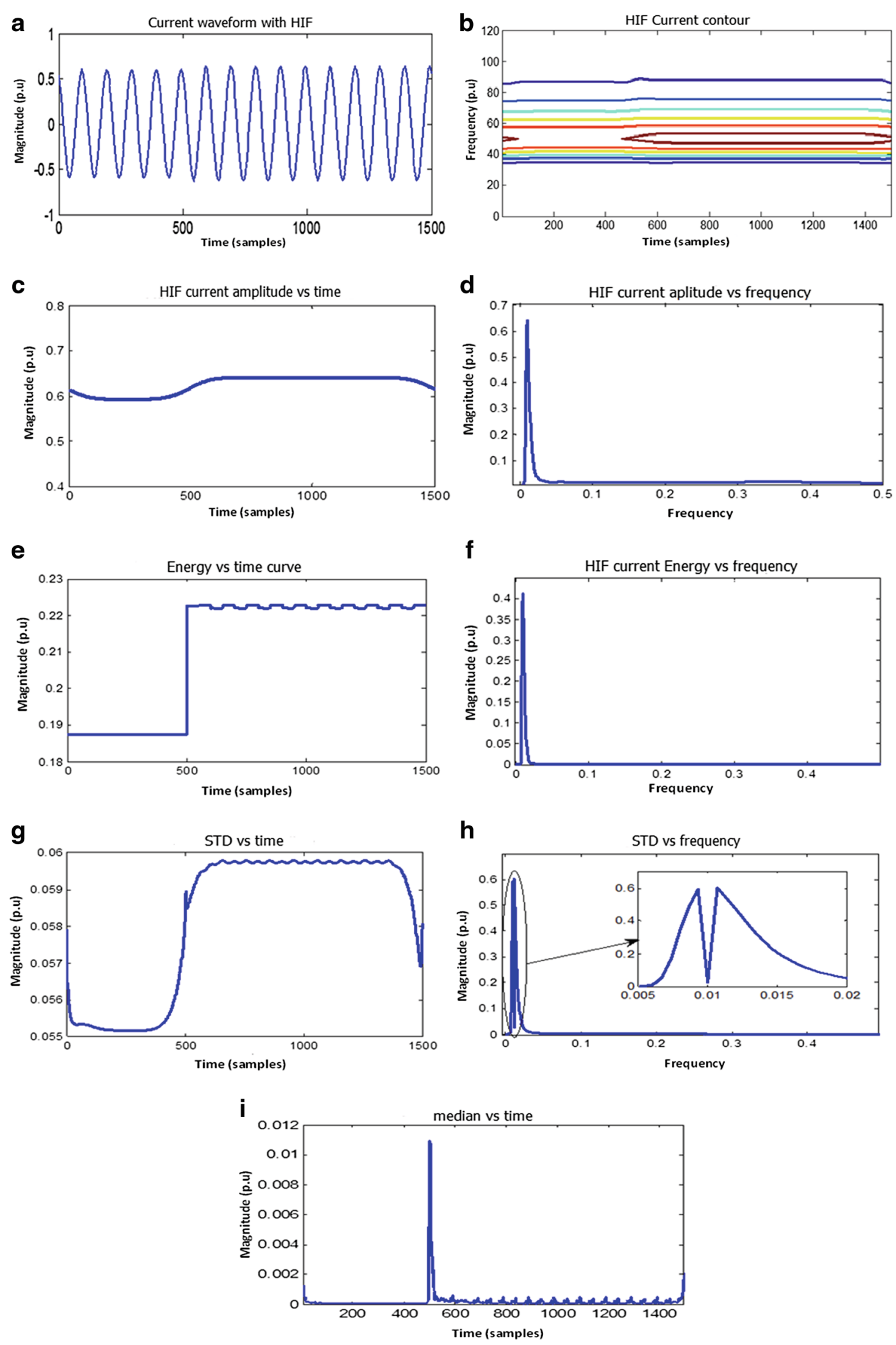

Fig. 6 a B-ph current signal with HIF b TF-contour c MAT-plot d MAF-plot e MET-plot $\mathbf{f}$ MEF-plot $\mathbf{g}$ STDT-plot h STDF-plot i median vs. Time plot 
Figure 6 shows the HIF signal, the time frequency, timeamplitude and frequency-amplitude plots generated from its STA matrix. Figure 6a shows the HIF current signal and Fig. $6 \mathrm{~b}$ shows the frequency versus time plot of STA which is called as time-frequency contour (TF-contour). It is clearly observed from the frequency contour that a substantial fluctuation occurred at the instant of HIF. Figure $6 \mathrm{c}$ shows the maximum amplitude vs. time plot (MAT-plot) that is obtained by searching columns of STA at each frequency. Figure $6 \mathrm{~d}$ gives the maximum amplitude vs. frequency plot (MAF-plot) that is maximum magnitude versus normalized frequency by searching rows of STA matrix. The plot shows the disturbance frequency components and their maximum amplitude. Figure 6e shows the energy vs. time plot (MET-plot) that gives the energy versus time by searching columns of STA at every frequency. Figure $6 f$ shows the energy vs. frequency plot (MEF-plot), that gives the energy versus frequency by searching row of STA. Figure $6 \mathrm{~g}$ shows the time-standard deviation plot (STDT-plot), which gives the standard deviation versus time by searching columns of STA at every frequency. Figure 6h shows the frequencystandard deviation plot (STDF-plot), that gives the standard deviation versus normalized frequency by searching rows of STA. Figure 6i shows the median vs. time plot of STA matrix.

Figure 7 shows a clear visual comparison between HIF and all other system conditions (normal fault, capacitor switching, load switching and no fault) based on energy with respect to time and frequency in Fig. 7a and b respectively. It is observed that the energy for normal fault condition is relatively higher than the other four conditions that can be easily distinguishable. In other four conditions, a little variation in the value of energy is observed but that can be accurately classified. Similarly Fig. 8 shows a complete visual comparison between HIF and all other conditions showing the magnitude vs. time plot (MAT-plot) and magnitude vs. frequency plot (MAF-plot) in Fig. 8a and $b$ respectively. Figure 9 shows a clear visual comparison between HIF and all other conditions based on the standard deviation vs. time plot (STDT-plot) and standard deviation vs. frequency plot (STDF-plot) in Fig. 9a and b respectively. Figure 10 shows a noticeable visual comparison between HIF and all other conditions based on the median vs. time plot. There is a sudden hike in the value of median approximately at 500 samples with respect to time.
Fig. 7 Comparison between HIF and all other system conditions based on Energy Vs Time and Frequency respectively in (a) and (b)
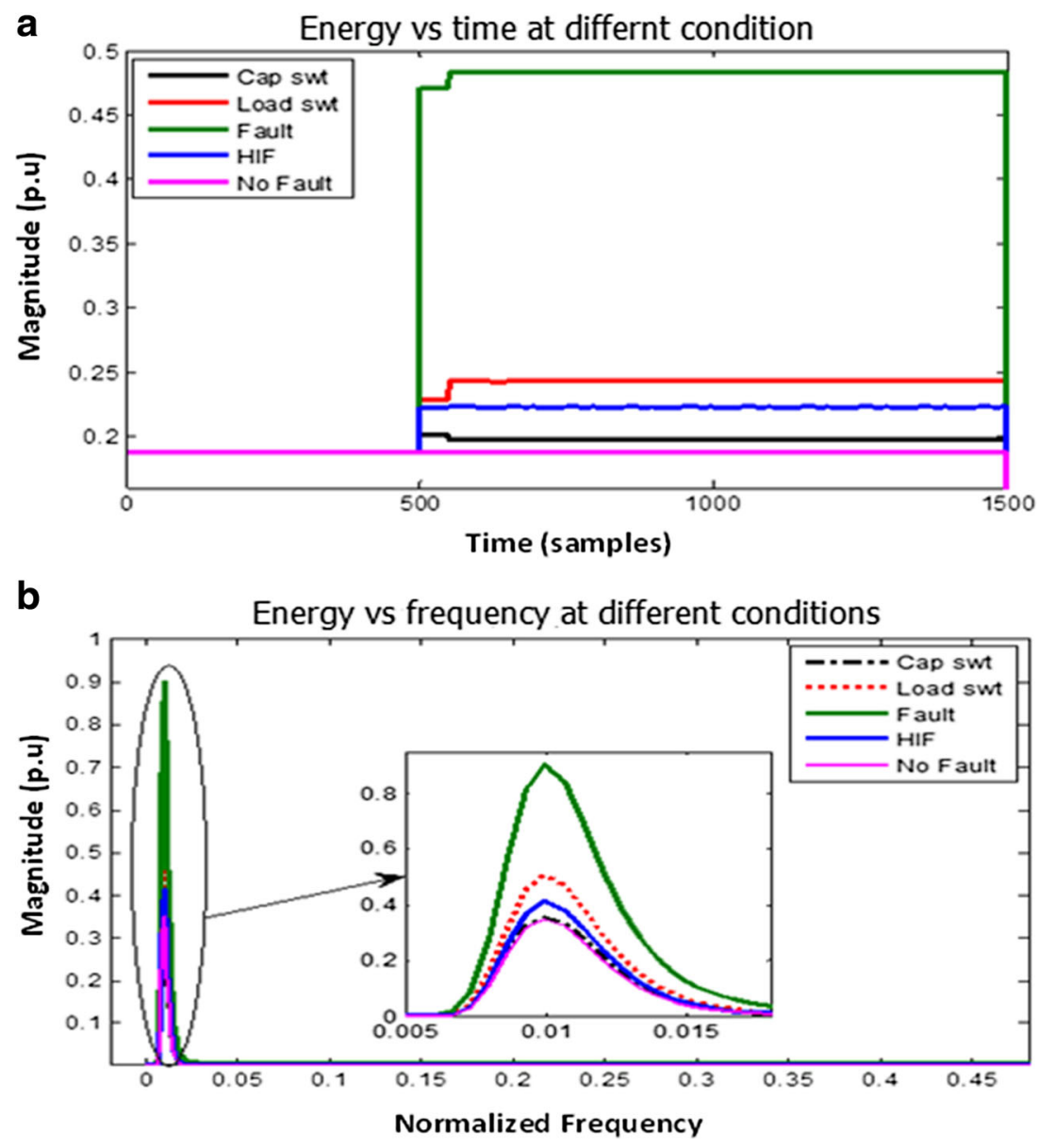
Fig. 8 Comparison between HIF and all other system conditions based on magnitude Vs time and frequency respectively in (a) and (b)

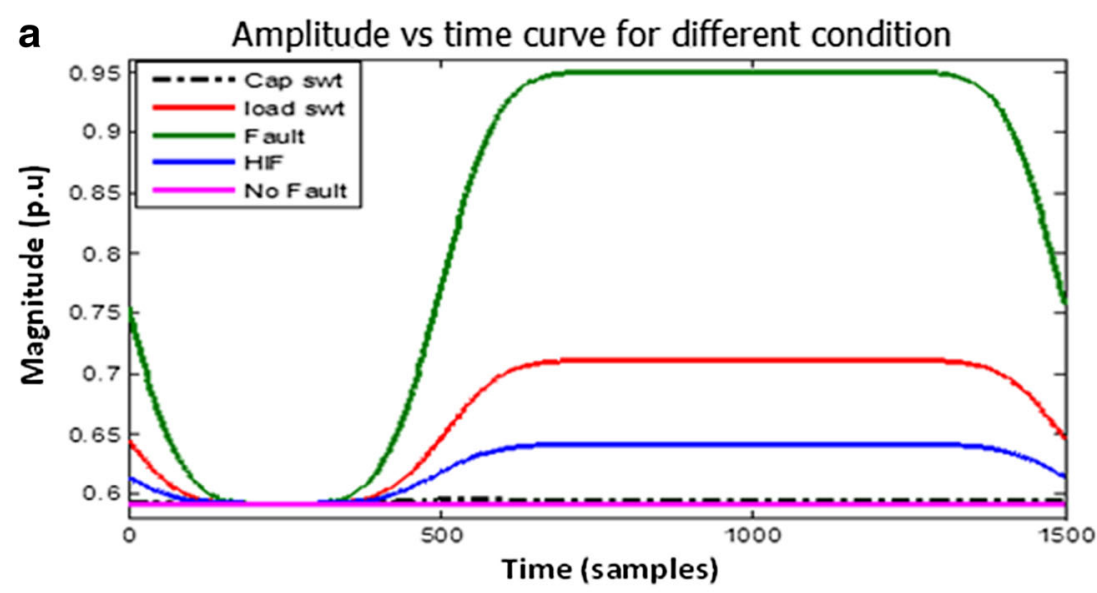

b Amplitude vs frequency curve for different condition

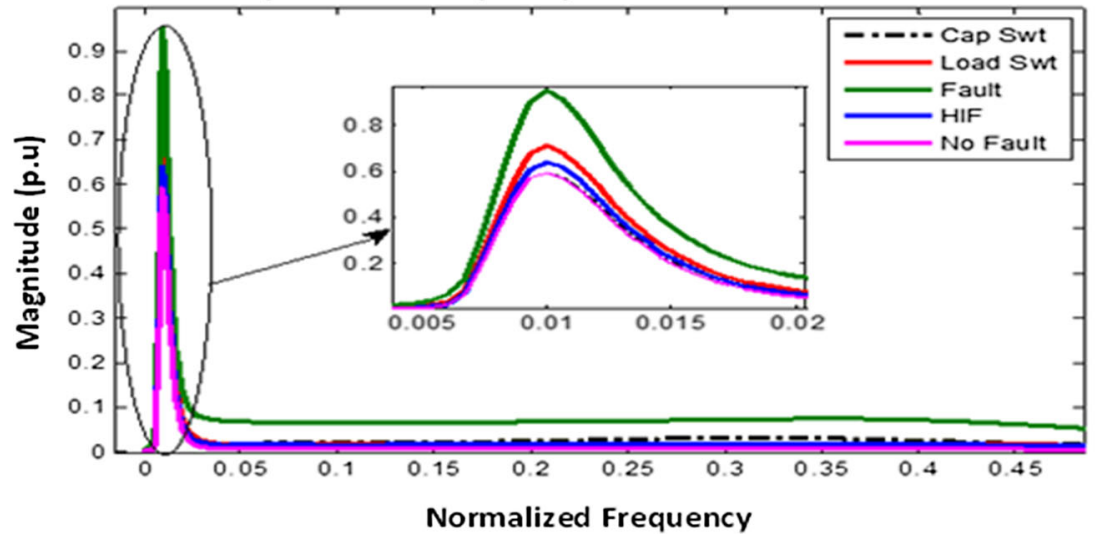

\section{Extracted Features}

S-transform is implemented to extract various features from generated current signals of all the proposed system conditions of power distribution networks. The time and frequency information of the resultant current signals is extracted from the generated S-matrix with accurate time and frequency resolution.

The time information is extracted from the S-matrix as:

$$
M A T=\max (a b s(S))
$$

Where MAT represents the maximum of the absolute value of the S-matrix (STA) generated from the S-transform which provides magnitude-time information.

Similarly frequency information is extracted using ST as:

$M A F=\max \left(a b s\left(S^{T}\right)\right)$

Where MAF states the maximum of the absolute value of the transposed STA generated using ST which provides magnitude-time(samples) information. Various features were extracted for each phases such as:

$F 1_{i}=A F=1-(\max (M A T)+\min (M A T))-F_{n}$

Where 'i' represents phases $(a, b, c)$
$F_{n}=\max (\mathrm{MAT})+\min (\mathrm{MAT})$ for no fault condition and $\mathrm{AF}$ is known as amplitude factor, represented as F1.

$F 2_{i}=\operatorname{entropy}(M A F)$

The entropy of magnitude-frequency plot (MAF-plot) is taken as feature 2 (F2).

$F 3_{i}=\max (M A F)$

Maximum value of magnitude-frequency plot (MAF-plot) is taken as feature $3(\mathrm{~F} 3)$.

$F 4_{i}=\max (M E T)$

Maximum value of Energy-time plot (MET-plot) is taken as feature $4(\mathrm{~F} 4)$.

Energy is calculated using the equation

$$
\begin{gathered}
M E T=\max \left(M A T^{2}\right) \\
F 5_{i}=\max (M A T)
\end{gathered}
$$

Maximum value of magnitude-time plot (MAT-plot) is taken as feature 5 (F5).

$F 6_{i}=\max (M S R(S T D F))$ 
Fig. 9 Comparison between HIF and all other system conditions based on standard deviation Vs. Time and Frequency respectively in (a) and (b)
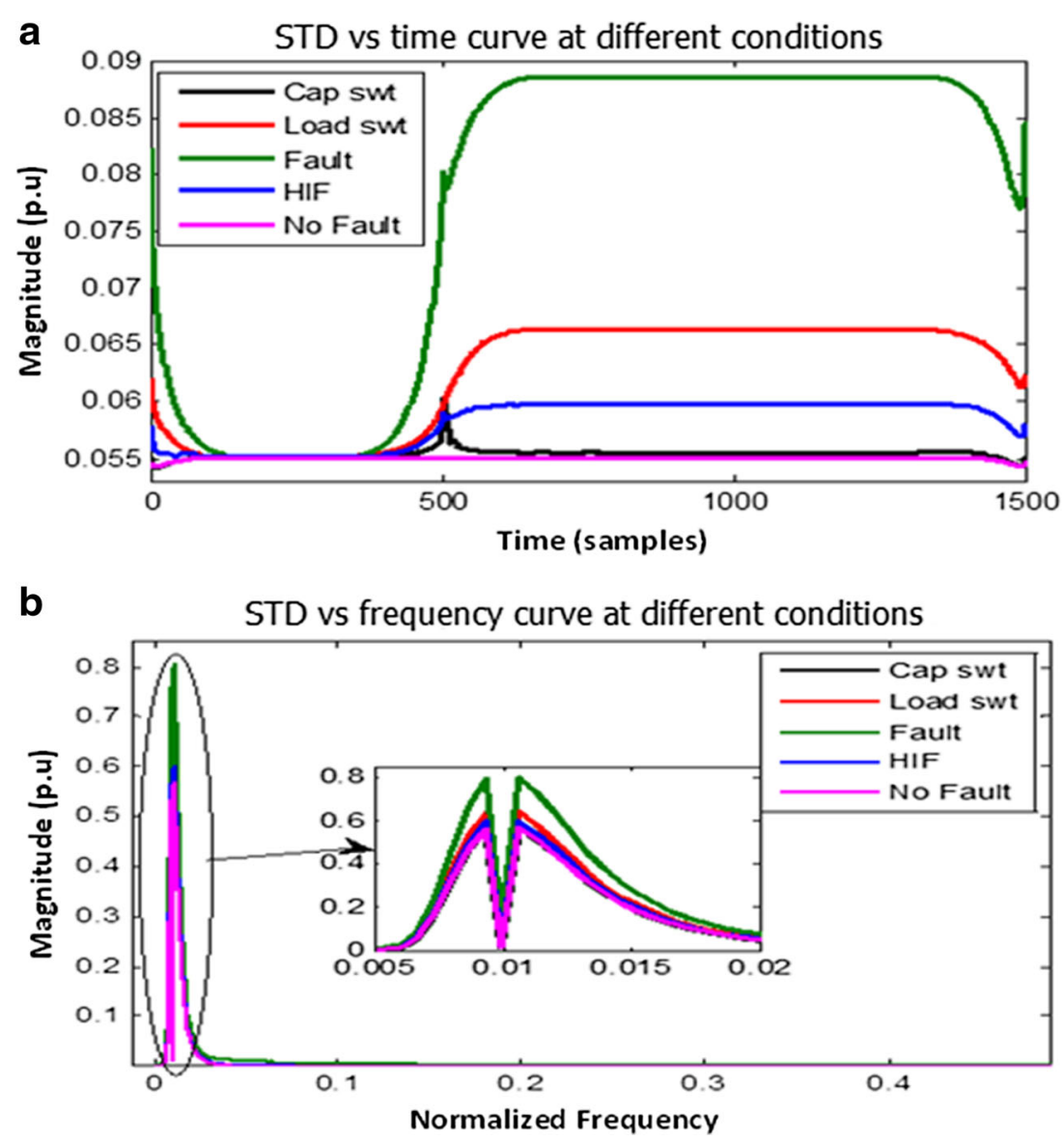

Feature 6 (F6) represents mean square root (MSR) of standard deviation of magnitude-frequency plot (STDF)

$$
F 7_{i}=S T D F=\operatorname{std}(M A F)
$$

Feature 7 (F7) gives standard deviation of MAF

$$
F 8_{i}=S T D T=\operatorname{std}(M A T)
$$

Feature 8 (F8) represents the value of standard deviation of magnitude-time plot (STDT)

$F 9_{i}=\max \left(R_{i}\right)$

Feature 9 (F9) represents the maximum value of the rate of change of energy (R) at each sample with respect to its previous sample.

Fig. 10 Median vs. Time plot

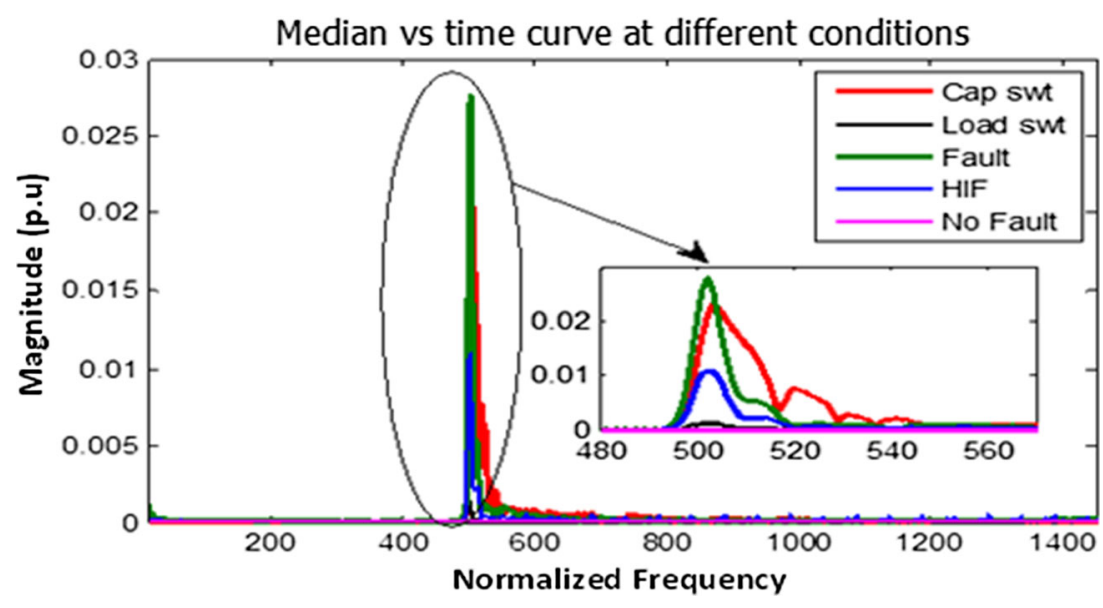


Fig. 11 Flowchart of Proposed method

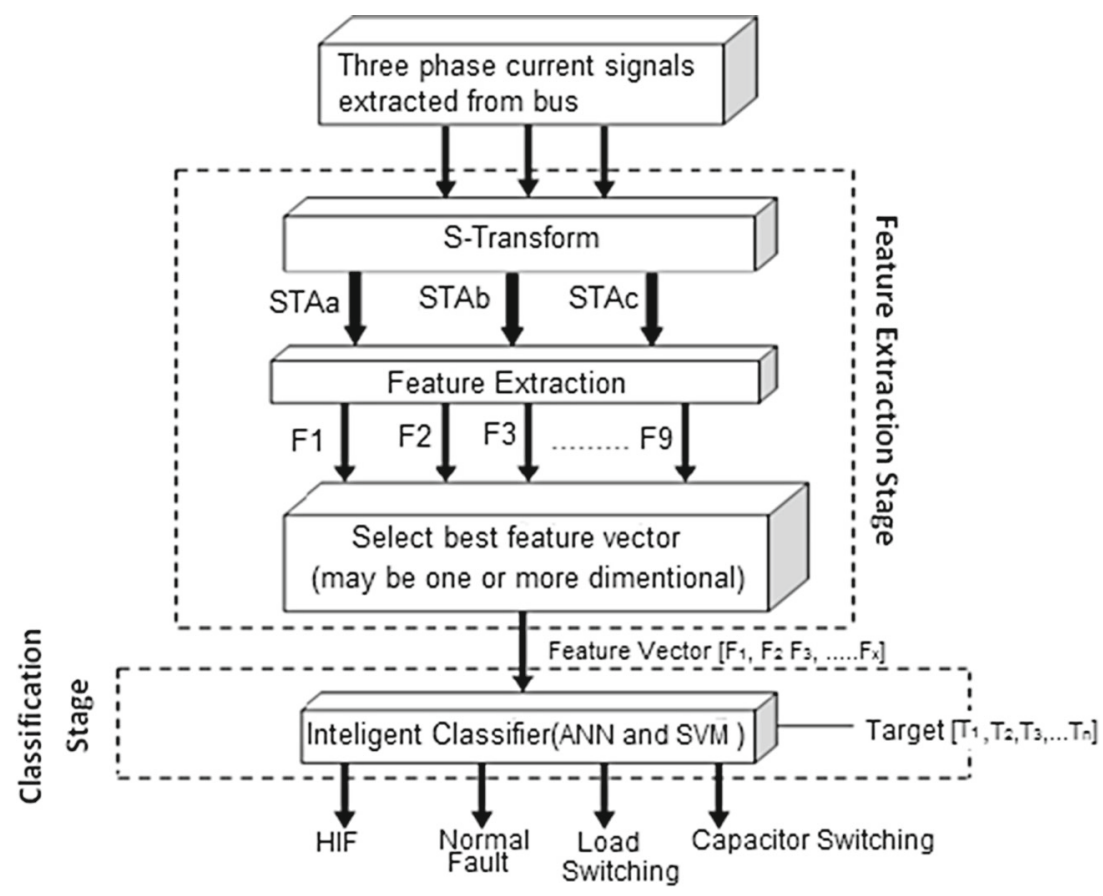

\section{Classification Of HIF}

The total number of 4000 different HIF and non-HIF (capacitor switching, load switching and normal fault) cases are simulated to extract features from respective current signals. These data are used as features which are extracted from ST technique and are used in the detection of HIF events through the proposed pattern recognition technique. The flowchart of the proposed technique is shown in Fig. 11.

\section{A. Result ANN classifier using input as ST Feature}

In this paper, the HIF classification process is realized with multi-layer perceptron (MLP) neural network, where resilient back propagation (RPROP) is

Table 1 Performance of ANN with single and combination of two features vector

\begin{tabular}{|c|c|c|c|c|c|c|c|c|}
\hline \multirow[t]{3}{*}{ Features vector } & \multicolumn{4}{|c|}{ Over all Accuracy (\%) For System I } & \multicolumn{4}{|c|}{ Over all Accuracy (\%) For System II } \\
\hline & \multirow[t]{2}{*}{ Normal condition } & \multicolumn{3}{|c|}{ Noisy condition } & \multirow[t]{2}{*}{ Normal condition } & \multicolumn{3}{|c|}{ Noisy condition } \\
\hline & & $50 \mathrm{db}$ & $40 \mathrm{db}$ & $30 \mathrm{db}$ & & $50 \mathrm{db}$ & $40 \mathrm{db}$ & $30 \mathrm{db}$ \\
\hline F1 & $95.50 \%$ & $88.30 \%$ & $85.10 \%$ & $82.10 \%$ & $95.50 \%$ & $88.80 \%$ & $86.90 \%$ & $83.80 \%$ \\
\hline $\mathrm{F} 2$ & $94.40 \%$ & $92.30 \%$ & $70.60 \%$ & $54.40 \%$ & $92.40 \%$ & $87.90 \%$ & $72.25 \%$ & $58.80 \%$ \\
\hline F3 & $94.40 \%$ & $93.90 \%$ & $88.53 \%$ & $86.50 \%$ & $94.40 \%$ & $86.50 \%$ & $81.35 \%$ & $76.50 \%$ \\
\hline F4 & $95.00 \%$ & $91.40 \%$ & $90.40 \%$ & $90.20 \%$ & $99.40 \%$ & $95.80 \%$ & $90.45 \%$ & $82.10 \%$ \\
\hline F5 & $94.80 \%$ & $93.60 \%$ & $90.20 \%$ & $87.20 \%$ & $96.20 \%$ & $91.80 \%$ & $89.80 \%$ & $83.80 \%$ \\
\hline $\mathrm{F} 6^{*}$ & $98.75 \%$ & $96.40 \%$ & $94.06 \%$ & $92.60 \%$ & $95.65 \%$ & $93.60 \%$ & $72.25 \%$ & $51.80 \%$ \\
\hline F7 & $98.00 \%$ & $96.90 \%$ & $93.24 \%$ & $89.10 \%$ & $96.50 \%$ & $89.30 \%$ & $81.75 \%$ & $51.60 \%$ \\
\hline F8 & $97.10 \%$ & $90.70 \%$ & $90.30 \%$ & $89.30 \%$ & $98.10 \%$ & $95.20 \%$ & $91.45 \%$ & $87.10 \%$ \\
\hline F9 & $96.32 \%$ & $94.50 \%$ & $90.60 \%$ & $86.60 \%$ & $95.35 \%$ & $93.30 \%$ & $85.90 \%$ & $70.40 \%$ \\
\hline $\mathrm{F}^{*} \mathrm{~F} 9$ & $99.60 \%$ & $97.60 \%$ & $97.20 \%$ & $93.50 \%$ & $97.60 \%$ & $96.40 \%$ & $79.25 \%$ & $71.20 \%$ \\
\hline $\mathrm{F} 6^{*} \mathrm{~F} 7$ & $100.00 \%$ & $100.00 \%$ & $97.70 \%$ & $93.70 \%$ & $95.40 \%$ & $94.40 \%$ & $78.35 \%$ & $51.40 \%$ \\
\hline $\mathrm{F} 6^{*} \mathrm{~F} 8$ & $99.25 \%$ & $99.20 \%$ & $98.20 \%$ & $94.10 \%$ & $96.35 \%$ & $96.35 \%$ & $91.45 \%$ & $70.45 \%$ \\
\hline F7 f8 & $100.00 \%$ & $98.50 \%$ & $97.62 \%$ & $91.60 \%$ & $95.00 \%$ & $92.45 \%$ & $82.35 \%$ & $65.45 \%$ \\
\hline F8 f9 & $99.55 \%$ & $98.75 \%$ & $93.57 \%$ & $89.75 \%$ & $100.00 \%$ & $97.11 \%$ & $92.45 \%$ & $81.00 \%$ \\
\hline
\end{tabular}

*An input to ANN results into higher accuracy then other single features vector 
Table 2 Overall performance of ANN with input feature F6 for system I

\begin{tabular}{|c|c|c|c|c|c|c|c|c|c|}
\hline \multirow{3}{*}{\multicolumn{2}{|c|}{ Signal }} & \multicolumn{4}{|c|}{ Normal Condition } & \multicolumn{4}{|c|}{ Noisy Condition } \\
\hline & & \multirow{2}{*}{\multicolumn{2}{|c|}{ Total cases }} & & & $50 \mathrm{~dB}$ & \multicolumn{2}{|c|}{$40 \mathrm{~dB}$} & \multirow[t]{2}{*}{$30 \mathrm{~dB}$} \\
\hline & & & & \multicolumn{2}{|c|}{ Correctly classified } & & & & \\
\hline \multicolumn{2}{|l|}{ HIF } & \multicolumn{2}{|c|}{700} & \multicolumn{2}{|c|}{$680(97.1 \%)$} & $678(96.8 \%)$ & \multicolumn{2}{|c|}{$677(96.7 \%)$} & $675(96.4 \%)$ \\
\hline \multirow[t]{3}{*}{ Non-HIF } & Normal Fault & 70 & & \multicolumn{2}{|c|}{$700(100 \%)$} & $700(100 \%)$ & \multicolumn{2}{|c|}{$695(88.8 \%)$} & $696(99.42 \%)$ \\
\hline & Capacitor Switching & 10 & & \multicolumn{2}{|c|}{$100(100 \%)$} & $65(65 \%)$ & \multicolumn{2}{|c|}{$43(43 \%)$} & $31(31 \%)$ \\
\hline & Load Switching & 10 & & \multicolumn{2}{|c|}{$100(100 \%)$} & $100(100 \%)$ & \multicolumn{2}{|c|}{$90(90 \%)$} & $80(80 \%)$ \\
\hline \multicolumn{2}{|c|}{ Average Accuracy } & & & \multicolumn{2}{|c|}{$1580(98.7 \%)$} & $1545(96.4 \%)$ & \multicolumn{2}{|c|}{$1376(94.06 \%)$} & $1158(92.6 \%)$ \\
\hline \multirow{7}{*}{\multicolumn{2}{|c|}{$\begin{array}{l}\text { Table } 3 \text { The classification } \\
\text { accuracy in terms of confusion } \\
\text { matrix having feature vector F6 } \\
\text { with } 50 \mathrm{db} \text { noisy condition for } \\
\text { system I }\end{array}$}} & \multirow{2}{*}{\multicolumn{2}{|c|}{ Signal }} & & HIF & \multicolumn{4}{|l|}{ Non-HIF } \\
\hline & & & & & & Normal Fault & Cap & Switching & Load Switching \\
\hline & & \multicolumn{3}{|l|}{ HIF } & 678 & 0 & \multicolumn{2}{|l|}{35} & 0 \\
\hline & & \multirow[t]{3}{*}{ Non-HIF } & \multicolumn{2}{|c|}{ Normal Fault } & 0 & 700 & \multicolumn{2}{|l|}{0} & 0 \\
\hline & & & \multicolumn{2}{|c|}{ Capacitor Switching } & 0 & 0 & \multicolumn{2}{|l|}{65} & 0 \\
\hline & & & \multicolumn{2}{|c|}{ Load Switching } & 22 & 0 & 0 & & 100 \\
\hline & & \multicolumn{3}{|c|}{ Average Accuracy } & $96.4 \%$ & & & & \\
\hline $\begin{array}{l}\text { Table } 4 \text { T } \\
\text { MLP used }\end{array}$ & $\begin{array}{l}\text { rchitecture of } \\
\text { ingle feature }\end{array}$ & Architect & & The & mber of & layers & & 3 & \\
\hline vector & & & & The & mber of & neuron on the la & & Input: $3, \mathrm{H}$ & den: 8 , Output: 4 \\
\hline & & & & The & tial weig & hts and biases & & Random & \\
\hline & & & & Acti & ion func & tions & & Tansig Tar & g Logsig \\
\hline & & Training & ameters & Lear & g rule & & & Resilient $\mathrm{b}$ & k propagation \\
\hline & & & & Lear & g rate & & & 0.5 & \\
\hline & & & & Mon & it consta & & & 0.98 & \\
\hline & & & & Mea & quared & error & & $1 \mathrm{e}-05$ & \\
\hline
\end{tabular}

Table 5 Performance of SVM with single and combination of two features vector

\begin{tabular}{|c|c|c|c|c|c|c|c|c|}
\hline \multirow[t]{3}{*}{ Features vector } & \multicolumn{4}{|c|}{ Over all Accuracy (\%) For System I } & \multicolumn{4}{|c|}{ Over all Accuracy (\%) For System II } \\
\hline & \multirow[t]{2}{*}{ Normal condition } & \multicolumn{3}{|c|}{ Noisy condition } & \multirow[t]{2}{*}{ Normal condition } & \multicolumn{3}{|c|}{ Noisy condition } \\
\hline & & $50 \mathrm{db}$ & $40 \mathrm{db}$ & $30 \mathrm{db}$ & & $50 \mathrm{db}$ & $40 \mathrm{db}$ & $30 \mathrm{db}$ \\
\hline F1 & $97.62 \%$ & $95.75 \%$ & $94.18 \%$ & $93.87 \%$ & $98.63 \%$ & $96.06 \%$ & $92.20 \%$ & $80.44 \%$ \\
\hline $\mathrm{F} 2$ & $96.81 \%$ & $91.19 \%$ & $72.00 \%$ & $56.24 \%$ & $95.02 \%$ & $94.88 \%$ & $70.18 \%$ & $49.31 \%$ \\
\hline F3 & $97.81 \%$ & $95.31 \%$ & $95.56 \%$ & $92.94 \%$ & $96.25 \%$ & $95.19 \%$ & $89.50 \%$ & $82.45 \%$ \\
\hline $\mathrm{F} 4$ & $100 \%$ & $99.06 \%$ & $96.25 \%$ & $92.25 \%$ & $99.88 \%$ & $98.44 \%$ & $94.25 \%$ & $85.89 \%$ \\
\hline F5 & $96.13 \%$ & $95.19 \%$ & $94.19 \%$ & $89.50 \%$ & $95.88 \%$ & $95.31 \%$ & $89.25 \%$ & $82.13 \%$ \\
\hline F6 & $100 \%$ & $100.00 \%$ & $94.06 \%$ & $92.13 \%$ & $100.00 \%$ & $98.13 \%$ & $94.32 \%$ & $86.25 \%$ \\
\hline F7 & $99.94 \%$ & $99.75 \%$ & $96.45 \%$ & $91.81 \%$ & $100.00 \%$ & $97.69 \%$ & $92.32 \%$ & $83.45 \%$ \\
\hline F8 & $96.31 \%$ & $96.19 \%$ & $94.25 \%$ & $91.56 \%$ & $94.88 \%$ & $95.56 \%$ & $88.31 \%$ & $66.44 \%$ \\
\hline F9 & $100 \%$ & $99.94 \%$ & $92.37 \%$ & $89.44 \%$ & $100.00 \%$ & $84.94 \%$ & $77.37 \%$ & $68.14 \%$ \\
\hline F6 F9 & $100 \%$ & $99.94 \%$ & $93.18 \%$ & $91.50 \%$ & $100.00 \%$ & $97.25 \%$ & $93.20 \%$ & $79.78 \%$ \\
\hline F6 F7 & $100 \%$ & $100.00 \%$ & $97.44 \%$ & $91.44 \%$ & $100.00 \%$ & $97.31 \%$ & $95.48 \%$ & $85.45 \%$ \\
\hline F6 F8 & $100 \%$ & $100.00 \%$ & $97.25 \%$ & $92.25 \%$ & $100.00 \%$ & $97.44 \%$ & $90.25 \%$ & $75.48 \%$ \\
\hline F7 f8 & $100 \%$ & $99.94 \%$ & $97.69 \%$ & $92.31 \%$ & $100.00 \%$ & $97.06 \%$ & $92.88 \%$ & $83.25 \%$ \\
\hline F8 f9 & $100 \%$ & $98.13 \%$ & $95.31 \%$ & $93.18 \%$ & $100.00 \%$ & $97.44 \%$ & $89.37 \%$ & $66.25 \%$ \\
\hline
\end{tabular}


Table 6 Overall performance of ANN with input feature F6 for system I

\begin{tabular}{|c|c|c|c|c|c|c|}
\hline \multirow[t]{3}{*}{ Signal } & & \multicolumn{2}{|c|}{ Normal Condition } & \multicolumn{3}{|c|}{ Noisy Condition } \\
\hline & & \multirow[b]{2}{*}{ Total cases } & & $50 \mathrm{~dB}$ & $40 \mathrm{~dB}$ & $30 \mathrm{~dB}$ \\
\hline & & & Correctly classified & & & \\
\hline \multicolumn{2}{|l|}{ HIF } & 700 & $700(100 \%)$ & $700(100 \%)$ & $677(96.7 \%)$ & $656(93.71 \%)$ \\
\hline \multirow[t]{3}{*}{ Non-HIF } & Normal Fault & 700 & $700(100 \%)$ & $700(100 \%)$ & $650(92.85 \%)$ & $666(95.15 \%)$ \\
\hline & Capacitor Switching & 100 & $100(100 \%)$ & $100(100 \%)$ & $81(81 \%)$ & $61(61 \%)$ \\
\hline & Load Switching & 100 & $100(100 \%)$ & $100(100 \%)$ & $97(97 \%)$ & $90(90 \%)$ \\
\hline \multicolumn{2}{|c|}{ Average Accuracy } & 1600 & $1600(100 \%)$ & $1600(100 \%)$ & $1505(94.06 \%)$ & $1473(92.13 \%)$ \\
\hline
\end{tabular}

Table 7 The classification accuracy in terms of confusion matrix having feature vector F6 with $30 \mathrm{db}$ noisy condition for system I

Table 8 Comparison of proposed method with WT methods

Table 9 Comparison of proposed method with existing methods

\begin{tabular}{llllll}
\hline Signal & HIF & \multicolumn{2}{l}{ Non-HIF } & & \\
\cline { 5 - 6 } & & & Normal Fault & Capacitor Switching & Load Switching \\
\hline HIF & & 656 & 0 & 16 & 10 \\
Non-HIF & Normal Fault & 0 & 666 & 23 & 0 \\
& Capacitor Switching & 0 & 34 & 61 & 0 \\
& Load Switching & 44 & 0 & 0 & 90 \\
\multirow{2}{*}{ Average Accuracy } & $92.13 \%$ & & \\
\hline
\end{tabular}

\begin{tabular}{|c|c|c|c|c|}
\hline \multirow[t]{2}{*}{ Classification Techniques (System I) } & \multicolumn{2}{|c|}{ Normal Condition } & \multicolumn{2}{|c|}{ With SNR 30dB } \\
\hline & WT & $\mathrm{ST}$ & WT & ST \\
\hline ANN & $100 \%$ & $100.00 \%$ & $85.6 \%$ & $93.70 \%$ \\
\hline SVM & $100 \%$ & $100 \%$ & $75.5 \%$ & $92.15 \%$ \\
\hline
\end{tabular}

\begin{tabular}{llcl}
\hline Relay & System Type & Ideal & \multicolumn{1}{c}{ SNR 30dB } \\
\hline Proposed Method & (max accuracy using best & Feature vector) & \\
ST feature with ANN & Redial & $100 \%$ & $93.70 \%$ \\
ST feature with SVM & Redial & $100 \%$ & $92.15 \%$ \\
ST feature with ANN & Mesh & $100 \%$ & $81.00 \%$ \\
ST feature with SVM & Mesh & $100 \%$ & $86.25 \%$ \\
Review paper/existing & & & \\
Ref. [29] & Redial & $93.6 \%$ & NA \\
Ref. [30] & Redial & $97.3 \%$ & NA \\
Ref. [5] & Redial & $96.0 \%$ & NA \\
Ref. [12] & Redial & $100 \%$ & NA \\
\hline
\end{tabular}


used as learning algorithm. The test system undertaken for the study is shown in Fig. 1. Out of total data $60 \%$ are used for training and $40 \%$ are used for testing for the classifier.

Table 1 depicts the classification accuracy result for HIF and non-HIF events with and without noisy condition for one and the combination of two features vector. It is clearly seen that even with the reduced number of features the ANN gives an adequate performance classification. It is easily noticeable in Table 1 that by using feature vector F6 as an input to ANN results into higher accuracy then other single features vector. The overall performance of ANN using features vector F6 is shown in Table 2. It is evident from Table 2 that the overall accuracy for classification HIF with other transient events and normal fault is $98.75 \%, 96.4 \%$, $94.06 \%$ and $92.60 \%$ for normal (without noise), with SNR 50db,with SNR 40db and with SNR 30db respectively.

The confusion matrix ( $4 \times 4)$ is shown in Table 3 , where the diagonal element represents the correctly classified events and non-diagonal elements depicts the misclassification.

Taking an appropriate combination of two feature vector (F6F7), the overall accuracy of classification is also shown in Table 1, where it indicates the percentage accuracy is increased to $100 \%, 100 \%, 97.70 \%$ and $93.70 \%$ for normal and noisy condition with SNR (signal to noise ratio) $50 \mathrm{db}, 40 \mathrm{db}$ and $30 \mathrm{db}$ respectively. The result indicates an inherent performance of the technique to detect HIF under noisy condition. The architecture of the MLP used for training with a combination of two feature vector is shown in Table 4.

B. Result SVM classifier using input as ST Feature

The overall performances of SVM for HIF classification with respect to Non-HIFs are explained in Tables 5, 6 and 7. Table 5 depict the classification accuracy result for HIF and non-HIF events with and without noisy condition for one and the combination of two feature vector. It is clearly seen that even with the reduced number of features the ANN gives an adequate performance classification. It is easily noticeable in Table 5 that by using feature vector F6 as an input to SVM results in higher accuracy than other single features vector. The overall performance of SVM using features vector F6 is shown in Tables 6 and 7.

C. Comparison of proposed method with existing technique based on accuracy

This section provides a comparison view of proposed ST based method with the WT based method.
Here the fault current extracted from PCC is processed through WT, and different features like energy content, standard deviation and entropy of signal are computed to train and test the ANN and SVM to discriminate the HIF from other transient condition and normal fault.

From Table 8 , it can be observed that the accuracy using WT_ANN in $30 \mathrm{~dB}$ noisy condition is $85.6 \%$ where as in case of ST_ANN is $93.70 \%$ which proves that ST provides a better accuracy under noisy condition than WT. So it is preferable to implement stransform under noisy conditions to detect HIF. Table 9 demonstrates the comparison between the proposed stransform based technique, and existing/proposed HIF detection methods. The information related to SNR 30 $\mathrm{dB}$ case are not available in the reference paper $[5,12$, $29,30]$ and is shown as NA (not available) in Table 9. The table clearly reveals an inherent performance of the technique to detect high impedance fault under noisy condition

\section{Conclusion}

In this work a novel method based on s-transform and pattern techniques for HIF detection and classification is suggested. In this method the fault current extracted from PCC is processed through ST to extract time-and frequency information in term of STA matrix. For appropriate classification it is necessary to choose a suitable feature vector that can recognize the characteristics of signal. So statistical features vectors based on S-transform are retrieved. Then, the effectiveness of features is improved by selecting combination of optimum features vector. These features vector are used to train and test the intelligent classifier. ANN and SVM are used as intelligent classifier in this paper. Comparative result shows that the SVM based classifier is superior to ANN based classifier to detect HIF for noisy condition.

The scope of the paper is limited to the application of ST for feature extraction. Further, the performance of HIF detection can be improved through the application other fast version of discrete s-transform. In the classification stage, this work is limited to the use of ANN and SVM. So, the efficiency of HIF classification may enhance through the other new machine learning techniques like extreme learning machine(ELM), because of its fast response than SVM and ANN. The studied system can be further simulated by using of a time varying HIF model to test the performance of the proposed method. 


\section{Nomenclatures}

Symbol Notations

CWT

$\tau$

$\mathrm{d}$

$S(\tau, f)$

$\phi(t, f)$

$\xi$

$\mathrm{b}$

$\omega$

L

\section{References}

1. Tengdin J (1989) Detection of downed conductors on utility distribution System, IEEE PES Tutorial course 90EH0310-3PWR

2. Phinyomark A, Nuidod A, Phukpattaranont P, Limsakul C Feature extraction and reduction of wavelet transform coefficients for EMG pattern classification, Department of Electrical Engineering, Faculty of Engineering, Prince of Songkla University 15 Kanjanavanich Road, Kho Hong, Hat Yai, Songkhla, 90112, Thailand, 2012. No. 6(122)

3. Mamishev AV, Russell BD, Benner CL (1996) Analysis of high impedance faults using fractal techniques. IEEE Trans Power Syst 11(1):435-440

4. Mamishev AV, Russell BD, Benner CL (1996) Analysis of high impedance faults using fractal techniques. IEEE Trans Power Syst 11(1):435-440

5. Sedighi AR, Haghifam MR, Malik OP, Ghassemian MH (2005) High impedance fault detection based on wavelet transform and statistical pattern recognition. IEEE Trans Power Deliv 20(4):2414-2421

6. Yang M-T, Guan J-L, Jhy-Cherng G (2007) High impedance faults detection technique based on wavelet transform. Int J Electr Electron Commun Energy Scie Engineer 1(4)

7. Goswami JC (1999) Fundamentals of wavelets. Wiley, New York

8. Yang MT, Gu JC, Jeng CY, Kao WS (2004) Detection high impedance fault in distribution feeder using wavelet transform and artificial neural networks. In: Intematlonal Conference on Power System Technology - POWERCON 2004 Slngapore pp. 21-24

9. Huang S-J, Hsieh C-T (1999) High-Impedance Fault detection utilizing a morlet wavelet transform approach. IEEE Trans Power Delivery 14(4)

10. Lai TM, Snider LA, Lo E, Sutanto D (2005) High-impedance fault detection using discrete wavelet transform and frequency range and RMS conversion. IEEE Trans Power Deliv 20(1):397407

11. Kim CJ, Russell BD (1995) Analysis of distribution disturbances and Arcing faults using the crest factor. Elect Power Syst Res 35:141-148

12. Gautam S, Brahma SM (2013) Detection of high impedance fault in power distribution systems using mathematical morphology. IEEE Trans Power Syst 28(2)
13. Girgis AA, Chang W, Makram EB (1990) Analysis of high impedance fault generated signals using a Kalman filtering approach. IEEE Trans Power Deliv 5(4):1714-1724

14. Sheng Y (2004) Member, IEEE, and Steven Rovnyak, Decision tree based methodology for high impedance fault detection. IEEE Trans Power Deliv 19(2)

15. Bishop C (1995) Neural networks for pattern recognition. University, Oxford

16. Etemadi AH, Sanaye-Pasand M (2008) High-impedance fault detection using multi-resolution signal decomposition and adaptive neural fuzzy inference system. IET Gener Transm Distrib 2(1)

17. Stockwell RG, Mansinha L, Lowe RP (1996) Localization of the complex spectrum: the S-transform. IEEE Trans Signal Process 44(4):99-100

18. Sedighi AR, Haghifam M-R (2010) Simulation of high impedance ground fault in electrical power distribution systems. In: Power System Technology (POWERCON), 2010 IEEE International Conference on, pp. 1-7

19. Lai TM, Snider LA, Lo E (2003) Wavelet transform based relay algorithm for the detection of stochastic high impedance faults. In: International Conference on Power System Transient, in New Orland, pp. 1-6 IPTS

20. Michalik M, Rebizant W, Lukowicz M, Lee S-J. (2005) KANG: Wavelet transform approach to high impedance fault detection in MV networks. In: Proceedings of the 2005 IEEE PowerTech Conference, St. petersburg, Russia, CD-ROM, paper 73

21. Pinnegar CR, Mansinha L (2003) The S-transform with windows of arbitrary an varying shape. Geophysics 68(1):381

22. Samantaray SR, Panigrahi BK, Dash PK (2008) High impedance fault detectionin power distribution networks using timefrequency transform and probabilistic neural network. IET Gener Transm Distrib 2(2):261-2

23. Roy N, Bhattacharya K (2014) Signal-energy based fault classification of unbalanced network using s-transform and neural network. Int J Recent Trends in Engineer Tech 10(2)

24. Hu X, Li S, Yang Y (2015) Advanced machine learning approach for Lithium-Ion battery state estimation in electric vehicles

25. Haykin S (2009) Neural networks and learning machines, $3^{\text {rd }}$. Pearson Prentice Hall

26. Deepa SN (2006) Introduction to neural networks using MATLAB 6. 0. TMH Publishing company, New Delhi

27. Hu X, Sun F (2009) Fuzzy clustering based multi-model support vector regression state of charge estimator for lithium-ion battery of electric vehicle. In: Intelligent Human-Machine Systems and Cybernetics, 2009. IHMSC'09. IEEE International Conference on, vol. 1, pp. 392-396

28. Hsu C-W, Chang C-C, Lin C-J (2010) A practical guide to support vector classification. In: Department of Computer Science National Taiwan University, Taipei 106, Initial version: 2003 Last updated: April 15

29. Ghaderi A, Mohammadpour HA, Ginn HL, Dhin YJ (2015) HighImpedance fault detection in the distribution network using the Time-Frequency-Based algorithm. IEEE Trans Power Delivery 30(3)

30. Sarlak M, Shahrtash SM (2011) High impedance fault detection using combination of multi-layer perceptron neural networks based on multiresolution morphological gradient features of current waveform. IET Gen Transm Distrib 5(5):588-595 